\title{
Correction to: R-catcher, a potent molecular tool to unveil the arginylome
}

\author{
Taewook Seo ${ }^{1,2}$. Jihyo Kim ${ }^{1} \cdot$ Ho-Chul Shin ${ }^{3}$ Jung Gi Kim ${ }^{1,2}$. Shinyeong Ju ${ }^{4,7} \cdot$ Laxman Nawale $^{1,2} \cdot$ Goeun Han $^{1,2}$. \\ Hye Seon Lee ${ }^{3}$. Geul Bang ${ }^{5}$. Jin Young Kim ${ }^{5}$. Jeong Kyu Bang ${ }^{6}$ Kyung Ho Lee ${ }^{1}$. Nak-Kyun Soung ${ }^{1}$. \\ Joonsung Hwang ${ }^{1}$. Cheolju Lee ${ }^{4,7}$. Seung Jun $\mathrm{Kim}^{2,3} \cdot$ Bo Yeon Kim $^{1,2} \cdot$ Hyunjoo Cha-Molstad $^{1,2}$ (])
}

Accepted: 5 August 2021 / Published online: 27 September 2021

(c) Springer Nature Switzerland AG 2021

\section{Correction to: \\ Cellular and Molecular Life Sciences (2021) 78:3725-3741 \\ https://doi.org/10.1007/s00018-021-03805-x}

The author would like to correct the errors in the publication of the original article. The corrected details are given below.
- An envelope symbol was missing near the co-author name "Taewook Seo, Jihyo Kim, Ho-Chul Shin, Jung Gi Kim" near affiliation.

- In Table 1, the word in the footnote "PROX4" should read as "PRDX4". The correct Table 1 with footnote is given below.
The original article can be found online at https://doi.org/10.1007/ s00018-021-03805-x.

Seung Jun Kim

ksj@kribb.re.kr

$\square$ Bo Yeon Kim

bykim@kribb.re.kr

$\triangle$ Hyunjoo Cha-Molstad hcha@kribb.re.kr

1 Anticancer Agent Research Center, Korea Research Institute of Bioscience and Biotechnology, Ochang-eup, Cheongju-si, Chungcheongbuk-do 28116, Republic of Korea

2 Department of Biomolecular Science, KRIBB School, University of Science and Technology, Daejeon 34113, Republic of Korea
3 Disease Target Structure Research Center, Korea Research Institute of Bioscience and Biotechnology, Daejeon 34141, Republic of Korea

4 Center for Theragnosis, Korea Institute of Science and Technology, Seoul 02792, Republic of Korea

5 Research Center for Bioconvergence Analysis, Korea Basic Science Institute, Ochang 28116, Republic of Korea

6 Division of Magnetic Resonance, Korea Basic Science Institute, Ochang 28116, Republic of Korea

7 KHU-KIST Department of Converging Science and Technology, Kyung Hee University, Seoul 02447, Republic of Korea 
Table 1 Arginylated ER protein candidates

\begin{tabular}{|c|c|c|c|c|c|}
\hline Uniprot ID & Gene symbol & Name & Predicted P1' site (signalP) & & Location \\
\hline P27797 & CALR & Calreticulin & $\begin{array}{l}\text { MLLSVPLLLGLLGLAVAEPAVYFKEOFLOGOG- } \\
\text { WTSRWIES }\end{array}$ & E18 & $\mathrm{Er}$ \\
\hline P50454 & SERPINH1 & Serpin H1 & $\begin{array}{l}\text { MRSLLLLSAFCLLEAALAAEVKKPAAAAAPG- } \\
\text { TAEKLSPKA }\end{array}$ & A19 & $\mathrm{Er}$ \\
\hline P10909 & CLU & Clusterin & $\begin{array}{l}\text { MMKTLLLFVGLLLTWESGOVLGDQTVS- } \\
\text { ONELOEMSNOGSK }\end{array}$ & D23 & $\mathrm{Er}$ \\
\hline 096AY3 & FKBP10 & Peptidyl-prolyl cis-trans isomerase FKBP10 & $\begin{array}{l}\text { MFPAGPPSHSLLRLPLLOLLLLVVOAVGRGL- } \\
\text { GRASPAGGP }\end{array}$ & $\mathrm{R} 29$ & $\mathrm{Er}$ \\
\hline 013162 & PRDX4 & Peroxiredoxin-4 & $\begin{array}{l}\text { MEALPLLAATTPOHGRHRRLLLLPLLLFLLPA- } \\
\text { GAVOGWET }\end{array}$ & W38 & $\mathrm{Er}$ \\
\hline $\mathrm{P} 14625$ & HSP90B1 & Endoplasmin & $\begin{array}{l}\text { MRALWVLGLCCVLLTFGSVRADDEVOVOGT- } \\
\text { VEEOLGKSRE }\end{array}$ & D22 & $\mathrm{Er}$ \\
\hline P11021 & HSPA5 & Endoplasmic reticulum chaperone $\mathrm{BiP}$ & $\begin{array}{l}\text { MKLSLVAAMLLLLSAARAEEEOKKEOVGTV- } \\
\text { VGIOLGTTYS }\end{array}$ & E19 & $\mathrm{Er}$ \\
\hline P81605 & $\mathrm{OCO}$ & Oermicidin & $\begin{array}{l}\text { MRFMTLLFLTALAGALVCAYDPEAASAPGSGN- } \\
\text { PCHEASAA }\end{array}$ & Y20 & Secreted \\
\hline 008380 & LGALS3BP & Galectin-3-binding protein & $\begin{array}{l}\text { MTPPRLFWVWLLVAGTOGVNOGOMRLAOG- } \\
\text { GATNOGRVEIF }\end{array}$ & V19 & Secreted \\
\hline P23142 & FBLN1 & Fibulin-1 & $\begin{array}{l}\text { MERAAPSRRVPLPLLLLGGLALLAAGVOADV- } \\
\text { LLEACCAOG }\end{array}$ & D30 & Secreted \\
\hline
\end{tabular}

10 ER proteins are listed. SignalP was used to predict P1' sites. The predicted P1' site is shown in bold red letters

- Supplementary File: The text in the supplementary file contains red mark and it is now corrected
Publisher's Note Springer Nature remains neutral with regard to jurisdictional claims in published maps and institutional affiliations.

Supplementary Information The online version contains supplementary material available at https://doi.org/10.1007/s00018-021-03915-6. 